\title{
Rho parameters from odd and even chirality, thermal QCD sum rules
}

\author{
S. Mallik and Krishnendu Mukherjee \\ Saha Institute of Nuclear Physics, 1/AF, Bidhannagar, Calcutta-700064, India
}

\begin{abstract}
Like the even chirality correlation functions of, say, the two vector currents, one can also consider odd chirality correlation functions to write thermal QCD sum rules. They contain fewer nonperturbative corrections, at least to the leading order. Here we write such a sum rule for the correlation function of vector and tensor 'currents'. The odd and even chirality sum rules are taken together to evaluate the effective parameters of the $\rho$ meson to second order in temperature. To within errors, the results are consistent and reproduce the absence of shift in the $\rho$ meson mass to this order.
\end{abstract}

\section{INTRODUCTION}

The vacuum QCD sum rules [1], when extended to finite temperature [2], provide a simple means to study the properties of the QCD medium. Below the critical temperature, they relate the temperature dependence of hadron parameters to the thermal average of local operators [3 5 .

Generally the sum rules are written for correlation functions of two currents, which have the same chirality, eg, of two vector or two axial vector currents. But one may also consider correlation functions of two quark bilinears of opposite chiralities [6]. Then their operator product expansions will be dominated, in each dimension, by the operators of odd chirality, the contributions of even chirality ones being highly suppressed by a factor of (small) quark mass in their coefficient functions. As a result the leading nonperturbative corrections will be given by a single operator, namely, the quark condensate; new odd chirality operators appear only as non-leading terms.

Here we write down the sum rule for the correlation function of the vector current and the tensor 'current'. Then both the present vector-tensor sum rule and the two vector-vector sum rules derived earlier get contributions from the same set of intermediate states with $J^{P G}=1^{-+}$. We consider the subtracted sum rules, obtained by subtracting out the vacuum sum rules from the corresponding thermal ones. The only intermediate states with significant contributions are then the non-resonant $2 \pi$ state and the $\rho$-resonance. The three sum rules form a convenient set for evaluation $[7]$.

We confine our evaluation at low temperature $T$ to leading order, which is $T^{2}$ in the chiral limit. Generally the thermal sum rules are complicated by the presence of Lorentz non-scalar operators, in addition to the Lorentz scalars contributing to the vacuum sum rules [8]. But to order $T^{2}$ non-scalar operators cannot contribute to these sum rules. Thus to this order, the contributing operators are the same as at zero temperature, their vacuum expectation values being replaced by the thermal averages.

In Sec II we describe the kinematics and present the result of operator product expansion. In Sec III we saturate the sum rules with $2 \pi$ and $\rho$ states. In Sec IV the sum rules are evaluated for the parameters of the $\rho$ meson to order $T^{2}$ in the chiral limit. Finally in Sec $\mathrm{V}$ we discuss the different aspects of the thermal sum rules and compare our evaluation with earlier ones.

\section{SUM RULES}

We restrict here to the better known non-strange channels of unit isospin. The quark bilinears in this channel are,

$$
S^{a}(x), V_{\mu}^{a}(x), T_{\mu \nu}^{a}(x), A_{\mu}^{a}(x), P^{a}(x)=\bar{q}(x)\left(1, \gamma_{\mu}, \sigma_{\mu \nu}, \gamma_{\mu} \gamma_{5}, \gamma_{5}\right) \frac{\tau^{a}}{2} q(x)
$$

$q(x)$ being the field of the $u$ and $d$ quark doublet and $\tau^{a}$ the Pauli matrices. Note that $S^{a}(x), P^{a}(x)$ and $T_{\mu \nu}^{a}(x)$ are odd and $V_{\mu}^{a}(x)$ and $A_{\mu}^{a}(x)$ are even under $\gamma_{5}$ transformation on the quark field.

As an important example of odd chirality correlation functions, consider the thermal average of the time ordered $(T)$ product of the vector current and the tensor 'current', 


$$
\mathcal{T}_{\mu \alpha \beta}^{a b}(q)=i \int d^{4} x e^{i q \cdot x}\left\langle T\left(V_{\mu}^{a}(x) T_{\alpha \beta}^{b}(\mathrm{o})\right)\right\rangle
$$

Here the thermal average of an operator $O$ is denoted by $\langle O\rangle$,

$$
\langle O\rangle=\operatorname{Tr} e^{-\beta H} O / \operatorname{Tr} e^{-\beta H},
$$

where $H$ is the QCD Hamiltonian, $\beta$ is the inverse of the temperature $T$ and $T r$ denotes the trace over any complete set of states.

As usual, it is convenient for kinematics to restore Lorentz invariance by introducing the four-velocity $u_{\mu}$ of matter. Then we can build the Lorentz scalars, $\omega=u \cdot q$ and $\bar{q}=\sqrt{\omega^{2}-q^{2}}$, representing the time and the space components of $q_{\mu}$ in the matter rest frame, $u_{\mu}=(1,0,0,0)$ 9. We now choose the three independent kinematic covariants as,

$$
\begin{aligned}
P_{\mu \alpha \beta} & =\eta_{\mu \alpha} q_{\beta}-\eta_{\mu \beta} q_{\alpha}, \\
Q_{\mu \alpha \beta} & =q_{\mu}\left(q_{\alpha} u_{\beta}-q_{\beta} u_{\alpha}\right)-q^{2}\left(\eta_{\mu \alpha} u_{\beta}-\eta_{\mu \beta} u_{\alpha}\right), \\
R_{\mu \alpha \beta} & =u_{\mu}\left(q_{\alpha} u_{\beta}-q_{\beta} u_{\alpha}\right)-\omega\left(\eta_{\mu \alpha} u_{\beta}-\eta_{\mu \beta} u_{\alpha}\right) .
\end{aligned}
$$

The kinematic decomposition now reads,

$$
\mathcal{T}_{\mu \alpha \beta}^{a b}(q)=i \delta^{a b}\left(P_{\mu \alpha \beta} T_{1}+Q_{\mu \alpha \beta} T_{2}+R_{\mu \alpha \beta} T_{3}\right)
$$

where the invariant amplitudes $T_{1,2,3}$ are functions of $\omega$ and $q^{2}$. In all computations, however, we shall revert back to the matter rest frame.

Only the amplitude $T_{1}$ survives at zero temperature. As we shall see below, at finite temperature the leading contributions (to order $T^{2}$ ) are also contained in $T_{1}$. So, working to the leading order, we have only to write the sum rule for this amplitude.

The advantage with odd chirality correlation functions becomes evident from an enumeration of local operators. The unit operator corresponds to the perturbative result. The non-perturbative power corrections begin with operators of dimension three and four. At dimension three, we have $\bar{q} q$ and $\bar{q} \psi q$ belonging to odd and even chirality respectively. (The operator $\bar{q} \psi q$ actually cannot contribute for zero chemical potential.) At dimension four, we have $\operatorname{tr} G_{\mu \nu} G^{\mu \nu}, u^{\mu} \Theta_{\mu \nu}^{f} u^{\nu}$ and $u^{\mu} \Theta_{\mu \nu}^{g} u^{\nu}$. Here the gauge field strength $G_{\mu \nu}=g \frac{\lambda^{a}}{2} G_{\mu \nu}^{a}, \lambda^{a}(a=1, \cdots 8)$ are the $S U(3)$ Gell-Mann matrices and $g$ is the QCD coupling constant. The operators $\Theta_{\mu \nu}^{f, g}$ are the energy momentum tensors for the quarks and gluons. (Note that in the matter rest frame, the latter two are just the energy densities. Also the operator $\bar{q} u \cdot D q$ where $D_{\mu}$ is the covariant derivative, is of odd chirality, but it can be reduced to $\hat{m} \bar{q} \psi q$ by using the equation of motion for the quark field.) All these operators of dimension four are of even chirality. Thus up to dimension four, only the operator $\bar{q} q$ can contribute significantly to the power correction for an odd chirality correlation function. At dimension five, there is only one Lorentz scalar operator, $\bar{q} \sigma^{\mu \nu} G_{\mu \nu} q$. In addition there are several Lorentz non-scalar operators contributing at finite temperature [10].

There is a simple configuration space approach 11 to the operator product expansion giving the Wilson coefficients of all operators, both scalars and non-scalars [12,13]. Using this method and restricting to scalar operators, we get for the product under consideration,

$$
T V_{\mu}^{a}(x) T_{\alpha \beta}^{b}(0) \rightarrow \delta^{a b}\left(\eta_{\alpha \mu} x_{\beta}-\eta_{\beta \mu} x_{\alpha}\right)\left\{\frac{3 \hat{m}}{2 \pi^{4}} \frac{1}{\left(x^{2}-i \epsilon\right)^{3}} \mathbf{1}+\frac{1}{2 \pi^{2}} \frac{1}{\left(x^{2}-i \epsilon\right)^{2}} \bar{u} u+\frac{1}{24 \pi^{2}} \frac{1}{\left(x^{2}-i \epsilon\right)} O_{5}+\cdots\right\},
$$

where $O_{5}$ is the dimension five operator, $O_{5}=\bar{u} \sigma_{\mu \nu} G^{\mu \nu} u$, and the dots represent operators of still higher dimensions. We have assumed $S U(2)$ flavour symmetry; $\hat{m}$ is the degenerate mass of $u$ and $d$ quarks and $\bar{u} u=\bar{d} d=\frac{1}{2} \bar{q} q$. The Fourier transform gives for space-like momenta $\left(Q^{2}=-q^{2} \geq 0\right)$,

$$
\mathcal{T}_{\mu \alpha \beta}^{a b}(q) \rightarrow i \delta^{a b}\left(\eta_{\mu \alpha} q_{\beta}-\eta_{\mu \beta} q_{\alpha}\right)\left\{-\frac{3 \hat{m}}{8 \pi^{2}} \log \left(Q^{2} / \mu^{2}\right)-\frac{1}{Q^{2}}\langle\bar{u} u\rangle+\frac{1}{3\left(Q^{2}\right)^{2}}\left\langle O_{5}\right\rangle+\cdots\right\} .
$$

Here $\mu(\simeq 1 \mathrm{GeV})$ is the renormalisation scale.

We wish to include the renormalization effects on the operators $T_{\mu \nu}^{a}$ and $\bar{u} u$. The coefficient $C\left(Q^{2} / \mu^{2}, g(\mu)\right)$ of $\bar{u} u$ satisfies 14,

$$
\left(\mu \frac{\partial}{\partial \mu}+\beta(g) \frac{\partial}{\partial g}+\gamma_{1}-\gamma_{2}\right) C\left(Q^{2} / \mu^{2}, g(\mu)\right)=0
$$


where $\beta(g)=-b \frac{g^{3}}{(4 \pi)^{2}}, \quad b=9$. The anomalous dimensions $\gamma_{1}$ and $\gamma_{2}$ of the operators $T_{\mu \nu}^{a}$ and $\bar{u} u$ can be easily calculated to give $\gamma_{1}=d_{1} \frac{g^{2}}{(4 \pi)^{2}}$ and $\gamma_{2}=d_{2} \frac{g^{2}}{(4 \pi)^{2}}$ with $d_{1}=8 / 3$ and $d_{2}=-8$. The solution to (2.6) may be written as

$$
C\left(Q^{2} / \mu^{2}, g(\mu)\right)=a\left(Q^{2}\right) C\left(1, \bar{g}\left(Q^{2}\right)\right),
$$

where $C\left(1, \bar{g}\left(Q^{2}\right)\right)$ is the lowest order result obtained above and

$$
a\left(Q^{2}\right)=\left(\frac{\log \left(Q^{2} / \Lambda^{2}\right)}{\log \left(\mu^{2} / \Lambda^{2}\right)}\right)^{\left(d_{1}-d_{2}\right) / 2 b} .
$$

The strong interaction scale is $\Lambda \simeq 200 \mathrm{MeV}$.

The other element needed for the sum rule is the spectral representation for the correlation function. The $T$ product at finite temperature has the so-called Landau representation in the variable $q_{0}$ at fixed $|\vec{q}|[15]$. At points on the imaginary axis $\left(q_{0}^{2}=-Q_{0}^{2}, Q_{0}^{2}>0\right)$, the representation for the invariant amplitudes are, up to subtractions, given by

$$
T_{1,2,3}\left(q_{0}^{2},|\vec{q}|\right)=\int_{0}^{\infty} d q_{0}^{\prime^{2}} \frac{N_{1,2,3}\left(q_{0}^{\prime},|\vec{q}|\right)}{q_{0}^{\prime^{2}}+Q_{0}^{2}} .
$$

where

$$
N_{1,2,3}\left(q_{0},|\vec{q}|\right)=\pi^{-1} \operatorname{ImT}_{1,2,3}\left(q_{0}^{2},|\vec{q}|\right) \tanh \left(\beta q_{0} / 2\right) .
$$

\section{SATURATION}

In the channel under consideration, the $\rho$-meson dominates the absorptive part. To find this contribution we write the matrix elements of the currents,

$$
\left\langle 0\left|V_{\mu}^{a}\right| \rho^{b}(k)\right\rangle=\delta^{a b} F_{\rho} m_{\rho} \epsilon_{\mu}, \quad\left\langle 0\left|T_{\alpha \beta}^{a}\right| \rho^{b}(k)\right\rangle=i \delta^{a b} G_{\rho}\left(\epsilon_{\alpha} k_{\beta}-\epsilon_{\beta} k_{\alpha}\right) .
$$

Here $F_{\rho}$ and $G_{\rho}$ are two coupling constants and $m_{\rho}$ and $\epsilon_{\alpha}$ are the mass and the polarization vector of the $\rho$-meson. $F_{\rho}$ is measured by the electronic decay rate of $\rho^{0}, F_{\rho}=153 \mathrm{MeV}$ [16]. Though the value of $G_{\rho}$ is not available directly from experiment, it can be obtained from one of the $\mathrm{U}(6)$ symmetry relations for the wave functions of a quark-anti-quark pair [17]. Defining the pion decay constant $F_{\pi}$ by

$$
\left\langle 0\left|A_{\mu}^{a}\right| \pi^{b}(k)\right\rangle=i \delta^{a b} k_{\mu} F_{\pi}
$$

this relation is

$$
G_{\rho}=\frac{1}{2}\left(F_{\rho}+F_{\pi}\right) .
$$

As $F_{\pi}=93 \mathrm{MeV}$, we get $G_{\rho}=123 \mathrm{MeV}$. This value of $G_{\rho}$ is also obtained from the QCD sum rules for the correlation function of two tensor 'currents' [6]. Comparing (3.1) with the matrix element of the $\rho$-meson field operator $\rho_{\mu}^{a}(x)$, $\left\langle 0\left|\rho_{\mu}^{a}\right| \rho^{b}\right\rangle=\delta^{a b} \epsilon_{\mu}$, we get the operator relations,

$$
V_{\mu}^{a}(x)=m_{\rho} F_{\rho} \rho_{\mu}^{a}(x), \quad T_{\alpha \beta}^{a}(x)=G_{\rho}\left(\partial_{\alpha} \rho_{\beta}^{a}-\partial_{\beta} \rho_{\alpha}^{a}\right) .
$$

The absorptive parts are now given essentially by that of the $\rho$-meson propagator at finite temperature. Working in the real time formulation of the thermal field theory [18], it is the 11-component of the $2 \otimes 2$ matrix propagator. We get

$$
N_{1}\left(q^{2}\right)=m_{\rho} F_{\rho} G_{\rho} \delta\left(q^{2}-m_{\rho}^{2}\right), \quad N_{2}\left(q^{2}\right)=N_{3}\left(q^{2}\right)=0 .
$$

This calculation must be interpreted as one in the effective field theory at finite temperature, where loop corrections make the parameters $m_{\rho}, F_{\rho}$ and $G_{\rho}$ temperature dependent. (At finite temperature, each of the particle-current coupling constants in (3.1-2) has, in general, different temperature dependence for the time and space components of the currents 19. But this bifurcation takes place only in orders higher than $T^{2}$. So it does not concern us here.) 
At lower energies there is the contribution of non-resonant two-pion state. Although small compared to the $\rho$-meson contribution, it describes the interaction of the currents with the pions in the heat bath and may assume importance in the difference sum rules we shall consider below. We find this absorptive part by writing the field theoretic expression for the pion loop at finite temperature. The pionic content $J_{\mu}^{a}(x)$ of the quark vector current $V_{\mu}^{a}(x)$ is given, to lowest order, by

$$
V_{\mu}^{a}(x) \rightarrow J_{\mu}^{a}(x)=\epsilon^{a b c} \phi^{b}(x) \partial_{\mu} \phi^{c}(x) .
$$

However the pionic content $S_{\alpha \beta}^{a}(x)$ of the quark tensor 'current' $T_{\alpha \beta}^{a}(x)$ is not immediately known, as it is not a symmetry current. From its index structure it may be written as

$$
T_{\alpha \beta}^{a}(x) \rightarrow S_{\alpha \beta}^{a}(x)=c \epsilon^{a b c} \partial_{\alpha} \phi^{b}(x) \partial_{\beta} \phi^{c}(x),
$$

where we determine the constant $c$ by comparing the two divergences,

$$
\partial^{\alpha} T_{\alpha \beta}^{a}(x)=2 \hat{m} V_{\beta}^{a}(x)+\cdots, \quad \partial^{\alpha} S_{\alpha \beta}^{a}(x)=c m_{\pi}^{2} J_{\beta}^{a}(x)+\cdots,
$$

the dots standing for higher derivative terms. Like $V_{\mu}^{a}(x)$ and $J_{\mu}^{a}(x)$, we can also identify $T_{\alpha \beta}^{a}(x)$ and $S_{\alpha \beta}^{a}(x)$, giving $c=2 \hat{m} / m_{\pi}^{2}=-F_{\pi}^{2} /\langle 0|\bar{u} u| 0\rangle$, on using the Gell-Mann, Oakes and Renner relation [20].

With the pionic version of $V_{\mu}^{a}(x)$ and $T_{\mu \nu}^{a}(x)$, it is simple to evaluate (2.1) to lowest order as,

$$
\mathcal{T}_{\mu \alpha \beta}^{a b}(q)=-c \delta^{a b} \int \frac{d^{4} k}{(2 \pi)^{4}}(2 k-q)_{\mu}\left(q_{\alpha} k_{\beta}-q_{\beta} k_{\alpha}\right) \Delta_{11}(k) \Delta_{11}(k-q),
$$

where $\Delta_{11}$ is the 11 - component of the thermal pion propagator. Its absorptive part can be calculated in the same way as for the vector-vector correlation function [5]. As we need to write the sum rule for the amplitude $T_{1}$, we quote the results for this amplitude only. In the time-like region (superscript + ),

$$
N_{1}^{+}=\frac{c q^{2}}{128 \pi^{2}} \int_{-v}^{v} d x\left(v^{2}\left(q^{2}\right)-x^{2}\right)\left\{1+2 n\left(\left(|\vec{q}| x+q_{0}\right) / 2\right)\right\}, \quad \text { for } \quad q^{2} \geq 4 m_{\pi}^{2},
$$

while in the space-like region (superscript -),

$$
N_{1}^{-}=\frac{c q^{2}}{128 \pi^{2}} \int_{v}^{\infty} d x\left(v^{2}\left(q^{2}\right)-x^{2}\right)\left\{n\left(\left(|\vec{q}| x-q_{0}\right) / 2\right)-n\left(\left(|\vec{q}| x+q_{0}\right) / 2\right)\right\}, \quad \text { for } \quad q^{2} \leq 0
$$

Here we have defined the functions $v(z)=\sqrt{1-4 m_{\pi}^{2} / z}$ and $n(z)=\left(e^{\beta z}-1\right)^{-1}$.

For $|\vec{q}| \rightarrow 0$, they reduce to simple expressions. In the time-like region,

$$
N_{1}^{+}=\frac{c q_{0}^{2} v^{3}\left(q_{0}^{2}\right)}{96 \pi^{2}}\left\{1+2 n\left(q_{0} / 2\right)\right\}
$$

In the space-like region, $q_{o}^{2}=\lambda|\vec{q}|^{2}$, with $0 \leq \lambda \leq 1$. Thus in the limit $|\vec{q}| \rightarrow 0, N_{1}^{-}$and its contribution to the spectral representation (2.7) are zero.

We can now write a spectral sum rule by equating at a space-like point the operator product expansion (2.5) to the spectral representation (2.7) with $\rho$ and $2 \pi$-contributions. Taking Borel transform as usual to get rid of any subtraction constant and to improve convergence of the spectral integral, we arrive at the thermal QCD sum rule,

$$
\begin{aligned}
& m_{\rho}(T) F_{\rho}(T) G_{\rho}(T) e^{-m_{\rho}^{2}(T) / M^{2}}-\frac{F_{\pi}^{2}}{96 \pi^{2}\langle 0|\bar{u} u| 0\rangle} \int_{4 m_{\pi}^{2}}^{\infty} d s s e^{-s / M^{2}} v^{3}(s)(1+2 n(\sqrt{s} / 2)) \\
= & \frac{3 \hat{m}}{8 \pi^{2}} M^{2}-a\left(M^{2}\right)\langle\bar{u} u\rangle+\frac{1}{3 M^{2}}\left\langle O_{5}\right\rangle .
\end{aligned}
$$

\section{EVALUATION}

As already stated, we shall evaluate sum rules to order $T^{2}$ in the chiral limit. Since the continuum integral is $0\left(T^{4}\right)$, the odd chirality sum rule (3.9), after subtraction of the corresponding vacuum sum rule, simplifies to 


$$
m_{\rho}(T) F_{\rho}(T) G_{\rho}(T) e^{-m_{\rho}^{2}(T) / M^{2}}-m_{\rho} F_{\rho} G_{\rho} e^{-m_{\rho}^{2} / M^{2}}=-a\left(M^{2}\right) \overline{\langle\bar{u} u\rangle}+\frac{1}{3 M^{2}} \overline{\left\langle O_{5}\right\rangle}
$$

Here the bar over the thermal average indicates subtraction of the corresponding vacuum expectation value.

For the sake of consistent evaluation in a closed framework, we augment this sum rule with the two even chirality sum rules for the vector-vector correlation function derived earlier [5]. In the chiral limit and omitting terms of order higher than $T^{2}$, they become

$$
\begin{aligned}
F_{\rho}^{2}(T) e^{-m_{\rho}^{2}(T) / M^{2}}-F_{\rho}^{2} e^{-m_{\rho}^{2} / M^{2}}+I_{T}\left(M^{2}\right) & =-\frac{\pi}{4} \frac{\overline{\left\langle O_{6}\right\rangle}}{M^{4}}, \\
F_{\rho}^{2}(T) m_{\rho}^{2}(T) e^{-m_{\rho}^{2}(T) / M^{2}}-F_{\rho}^{2} m_{\rho}^{2} e^{-m_{\rho}^{2} / M^{2}} & =\frac{\pi}{2} \frac{\overline{\left\langle O_{6}\right\rangle}}{M^{2}}
\end{aligned}
$$

where

$$
I_{T}\left(M^{2}\right)=\frac{1}{24 \pi^{2}} \int_{0}^{\infty} d s\left(1+e^{-s / M^{2}}\right) \frac{1}{e^{\sqrt{s} / 2 T}-1}=\frac{T^{2}}{9}+0\left(T^{4}\right)
$$

and $O_{6}$ is the four-quark operator, $O_{6}=\alpha_{s} \bar{q} \gamma_{\mu} \gamma_{5} \lambda^{a} \tau^{3} q \bar{q} \gamma^{\mu} \gamma_{5} \lambda^{a} \tau^{3} q$ 21]. This operator was ignored in [5].

At low temperature the heat bath consists primarily of dilute pion gas. The thermal trace can then be approximated by the vacuum and the one pion state. The pion matrix element of the operator can be evaluated by using PCAC and soft pion technique [3]. One gets,

$$
\langle\bar{u} u\rangle=\langle 0|\bar{u} u| 0\rangle\left(1-\frac{T^{2}}{8 F_{\pi}^{2}}\right), \quad\left\langle O_{5}\right\rangle=\left\langle 0\left|O_{5}\right| 0\right\rangle\left(1-\frac{T^{2}}{8 F_{\pi}^{2}}\right), \quad\left\langle O_{6}\right\rangle=\left\langle 0\left|O_{6}\right| 0\right\rangle\left(1-\frac{T^{2}}{3 F_{\pi}^{2}}\right) .
$$

where the vacuum expectation values are all known 22, 24],

$$
\langle 0|\bar{u} u| 0\rangle=-(225 \mathrm{MeV})^{3}, \quad\left\langle 0\left|O_{5}\right| 0\right\rangle=m_{o}^{2}\langle 0|\bar{u} u| 0\rangle, \quad\left\langle 0\left|O_{6}\right| 0\right\rangle=6.5 \times 10^{-4} \mathrm{GeV}^{6} .
$$

with $m_{o}^{2}=1.0 \mathrm{GeV}^{2}$. If we write

$$
m_{\rho}(T)=m_{\rho}\left(1+a \frac{T^{2}}{F_{\rho}^{2}}\right), \quad F_{\rho}(T)=F_{\rho}\left(1+b \frac{T^{2}}{F_{\rho}^{2}}\right), \quad G_{\rho}(T)=G_{\rho}\left(1+c \frac{T^{2}}{F_{\rho}^{2}}\right),
$$

the sum rules (4.1-3) predict the values of the constants $a, b$ and $c$ as

$$
\begin{aligned}
& a=e^{m_{\rho}^{2} / M^{2}}\left\{\frac{1}{18}-\frac{K_{2}}{4 M^{4}}\left(1+\frac{2 M^{2}}{m_{\rho}^{2}}\right)\right\}, \\
& b=-e^{m_{\rho}^{2} / M^{2}}\left\{\frac{1}{18}\left(1-\frac{m_{\rho}^{2}}{M^{2}}\right)+\frac{K_{2}}{4 M^{4}}\left(1+\frac{m_{\rho}^{2}}{M^{2}}\right)\right\}, \\
& c=e^{m_{\rho}^{2} / M^{2}}\left\{\frac{m_{\rho}^{2}}{18 M^{2}}+K_{1}\left(a\left(M^{2}\right)-\frac{m_{0}^{2}}{3 M^{2}}\right)-\frac{K_{2}}{4 M^{4}}\left(\frac{m_{\rho}^{2}}{M^{2}}-\frac{2 M^{2}}{m_{\rho}^{2}}+2\right)\right\},
\end{aligned}
$$

where

$$
K_{1}=\frac{\langle 0|\bar{u} u| 0\rangle}{4 m_{\rho} F_{\pi}^{2}\left(1+F_{\pi} / F_{\rho}\right)}, \quad K_{2}=\frac{\pi}{6 F_{\pi}^{2}}\left\langle 0\left|O_{6}\right| 0\right\rangle
$$

It should be noted that the parameters $m_{\rho}, F_{\rho}$ and $F_{\pi}$ in eqn.(4.8-10) refer to their chiral limits and not the physical values. There does not appear to exist any calculation of $F_{\rho}$ to this limit in the literature [25]. Under this circumstance, let us evaluate $a, b$ and $c$ with the physical values of these parameters as quoted in Sec. III and hope that the results will not be affected much. 


\begin{tabular}{|c|c|c|c|}
\hline $\begin{array}{c}M^{2} \\
\left(\mathrm{GeV}^{2}\right)\end{array}$ & $a$ & $b$ & $c$ \\
\hline 0.8 & 0 & -0.086 & -0.21 \\
\hline 1.0 & 0.020 & -0.069 & -0.24 \\
\hline 1.2 & 0.035 & -0.063 & $-0 . .26$ \\
\hline 2.0 & 0.049 & -0.057 & -0.30 \\
\hline 4.0 & 0.054 & -0.056 & -0.33 \\
\hline & & & \\
\hline
\end{tabular}

Table 1: Coefficients of $T^{2}$ in $m_{\rho}(T), F_{\rho}(T)$ and $G_{\rho}(T)$ at different values of $M^{2}$.

Table I shows the evaluation of the constants $a, b$ and $c$ for different values of the Borel parameter $M^{2}$ over the range $0.8 \leq M^{2} \leq 4 \mathrm{in} \mathrm{GeV}^{2}$. It is seen that as $M^{2}$ increases, the values change but rather slowly. Observe that at $M^{2}=0.8 G e V^{2}$, one gets $a=0$, reproducing the result that the hadron masses do not shift to order $T^{2}$ [26, 27]. This suggests that we take the central values of all the constants at this value of $M^{2}$ in the prediction of our sum rules,

$$
a=0, \quad b=-.086, \quad c=-.21,
$$

with an error of about $25 \%$, estimated from their variations in the range of $M^{2}$ considered.

$\mathrm{Eq}(3.3)$ relating $G_{\rho}$ to $F_{\rho}$ and $F_{\pi}$ is used above only at zero temperature. Being a symmetry relation one expects it to be valid also at finite temperature,

$$
G_{\rho}(T)=\frac{1}{2}\left(F_{\rho}(T)+F_{\pi}(T)\right) .
$$

This equation provides another determination of $c$, if we take $F_{\rho}(T)$ as determined above and

$$
F_{\pi}(T)=F_{\pi}\left(1-\frac{T^{2}}{12 F_{\pi}^{2}}\right)
$$

as obtained from chiral perturbation theory. It gives $c=-.14$, in fair agreement with $c=-.21$ obtained above.

\section{DISCUSSION}

We derive here an odd chirality thermal QCD sum rule. More such sum rules can be written by considering other correlation functions of odd chirality, for example, of the axial vector and pseudo-scalar currents. Generally they bring in a different set of operator expectation values, but are fewer in number compared to those in the even chirality sum rules, at least to the leading order. It would be useful to consider both sets of sum rules for numerical evaluation.

The behaviour of the absorptive parts in the space-like region in different sum rules is worth mentioning here. Such absorptive parts are a characteristic feature of thermal field theories and here it describes pion absorption by the current from the heat bath and its emission into it. For nonzero $|\vec{q}|$, it is present in all the sum rules. However, as $|\vec{q}| \rightarrow 0$, it does or does not survive depending on the kinematics. In the sum rules considered here, it survives only in the sum rule (4.2), obtained from the longitudinal amplitude defined in Ref. [5].

We have evaluated the sum rules to order $T^{2}$ in the chiral limit. The sum rules are expected to be well saturated to this order and should be independent of the Borel parameter $M^{2}$ over a larger range. The subtraction eliminates the continuum contribution beyond about $1 \mathrm{GeV}$, it being practically independent of $T$ for $T<150 \mathrm{MeV}$, say. There are thus no other significant source of contribution in the spectral representation besides those already included, namely, the $2 \pi$-continuum and the $\rho$-resonance.

As regards the operators, only those which are Lorentz scalars and appear already in the corresponding vacuum sum rules, may produce terms of order $T^{2}$. We note, however, that while the $T^{2}$ contributions in the spectral representation arise only from $2 \pi$ and $\rho$ states, scalar operators of all dimensions can in general contribute to this order, though, of course, the usual Borel suppression operates for operators of higher dimensions. 
The other point to note is that the perturbative contribution, which is the coefficient of the unit operator, is eliminated by subtraction. So the higher dimension operators, which constituted only (power) corrections to the perturbative result in the vacuum sum rules, are now assuming the leading role. Thus the subtracted version of sum rules considered here brings out clearly the sensitivity of the thermal sum rules to any uncertainty in the operator expectation values.

The present work is a continuation of our earlier work [5]. There our main concern was to include all the operators up to dimension four with their renormalisation effects in the vector-vector sum rules. But the omission of the scalar, four quark operator was not justified. For, while the dimension four operators are significant at higher temperatures near the critical point, at low temperatures it is only this dimension six operator which dominates, contributing to order $T^{2}$ in the chiral limit.

The most extensive earlier work is that of Hatsuda et al [3]. They include contributions up to order $T^{6}$ from the operators and plot the $T$-dependence of $m_{\rho}(T)$ and $F_{\rho}(T)$ ( and also parameters of other resonances) but do not obtain the coefficients of $T^{2}, T^{4}$ etc. (Incidentally their evaluation up to order $T^{6}$ is incomplete, because they include contributions up to order $T^{4}$ and $T^{6}$ only when they are leading in some operators, but neglect non-leading contributions even to order $T^{4}$ in others, eg, $\langle\bar{u} u\rangle$.) Eletsky et al [28] calculate the $\rho$ meson mass to order $T^{4}$, but do not take the mixing of dimension four, Lorentz non-scalar operators under renormalisation.

[1] M.A. Shifman, A.I. Vainshtein and V.I. Zakharov, Nucl. Phys. B147, 385 (1979). For a collection of original papers and comments, see Vacuum Structure and QCD Sum Rules, edited by M.A. Shifman (North Holland, Amsterdam, 1992). See also S. Narison, QCD Spectral Sum Rules (World Scientific, Singapore, 1989).

[2] A.I. Bochkarev and M.E. Shaposhnikov, Nucl. Phys. B268, 220 (1986).

[3] T. Hatsuda, Y. Koike and S.H. Lee, Nucl. Phys. B394, 221 (1993). The earlier papers may be traced from this one.

[4] V.L. Eletsky and B.L. Ioffe, Phys. Rev. D 51, 2371 (1995).

[5] S. Mallik and K. Mukherjee, Phys. Rev. D 58, 096011 (1998).

[6] S. Mallik, Nucl. Phys. B206, 90 (1982).

[7] One might think of considering also the correlation function of two tensor currents. But the corresponding sum rules bring in the $J^{P G}=1^{++}$resonance also.

[8] E.V. Shuryak, Rev. Mod. Phys. 65, 1 (1993).

[9] H.A. Weldon, Phys. Rev. D 26, 1394 (1982).

[10] X. Jin, T.D. Cohen, R.J. Furnstahl and D.K. Griegel, Phys. Rev. C 47, 2882 (1993).

[11] H. Fritzsch and H. Leutwyler, Phys. Rev. D 10, 1624 (1974).

[12] W. Hubschmid and S. Mallik, Nucl. Phys. B207, 29 (1982).

[13] S. Mallik, Phys. Lett. B 416, 373 (1998).

[14] See, for example, M.E. Peskin and D.V. Schroeder, An Introduction to Quantum Field Theory (Addison-Wesley, 1995).

[15] L.D. Landau, Sov. Phys. -JETP 7, 182 (1958), reproduced in Collected Works of L.D. Landau, edited by D.Ter Haar (Pergamon Press, 1965). See also A.L. Fetter and J.D. Walecka, Quantum Theory of Many-particle Systems (McGraw-Hill Book Co., 1971).

[16] Particle Data Group, Phys. Rev. D 54, 1 (1996).

[17] H. Leutwyler, Nucl. Phys., B76, 413 (1974).

[18] A.J. Niemi and G.W. Semenoff, Ann. Phys. 152, 105 (1984). See also N.P. Landsman and Ch.G.van Weert, Phys. Rep. 145, 141 (1987).

[19] H. Leutwyler, Phys.Rev. D 49, 3033 (1994); R.D. Pisarski and M. Tytgat, Phys. Rev. D 54, 2989 (1996); D.Toublan, Phys. Rev. D56, 5629 (1997).

[20] M. Gell-Mann, R.J. Oakes and B. Renner, Phys. Rev., 175, 2195 (1968).

[21] The other scalar piece in $O_{6}$ does not contribute to order $T^{2}$.

[22] J. Gasser and H. Leutwyler, Phys. Lett. B 184, 83 (1987). P. Gerber and H. Leutwyler, Nucl. Phys, B321, 387

[23] V.M. Belyaev and B.L. Ioffe, Sov. Phys. JETP, 57, 721 (1983).

[24] A higher value has been suggested for $\left\langle 0\left|O_{6}\right| 0\right\rangle$ in S. Narison, QCD Spectral sum rules (World Scientific, Singapore, 1989). Again the vacuum saturation value becomes smaller with the present value for $\langle 0|\bar{u} u| 0\rangle$. Here we stick to the value originally obtained in [1].

[25] J. Bijnens, P. Gosdzinsky and P. Talavera, Nucl. Phys. B501, 495 (1997). They calculate the chiral limit of $m_{\rho}$ but not of $F_{\rho}$.

[26] H. Leutwyler and A.V. Smilga, Nucl. Phys., B342, 302 (1990).

[27] V.L. Eletsky and B.L. Ioffe, Phys. Rev. D47, 3083 (1993).

[28] V.L. Eletsky and B.L. Ioffe, Phys. Rev. D51, 2371 (1995). 